\title{
Invisible decay modes in nuclear gamma cascades
}

\author{
Giovanni Benato, ${ }^{1,2}$ Alexey Drobizhev, ${ }^{2}$ Surjeet Rajendran, ${ }^{3}$ and Harikrishnan Ramani ${ }^{3,4, *}$ \\ ${ }^{1}$ Department of Physics, University of California, Berkeley, California 94720, USA \\ ${ }^{2}$ Nuclear Science Division, Lawrence Berkeley National Laboratory, Berkeley, California 94720, USA \\ ${ }^{3}$ Berkeley Center for Theoretical Physics, Department of Physics, \\ University of California, Berkeley, California 94720, USA \\ ${ }^{4}$ Theoretical Physics Group, Lawrence Berkeley National Laboratory, Berkeley, California 94720, USA
}

(Received 28 November 2018; published 19 February 2019)

\begin{abstract}
We propose a high statistics experiment to search for invisible decay modes in nuclear gamma cascades. A radioactive source (such as ${ }^{60} \mathrm{Co}$ or ${ }^{24} \mathrm{Na}$ ) that triggers gamma cascades is placed in the middle of a large, hermetically sealed scintillation detector, enabling photon identification with high accuracy. Invisible modes are identified by establishing the absence of a photon in a well-identified gamma cascade. We propose the use of fast scintillators with nanosecond timing resolution, permitting event rates as high as $10^{7} \mathrm{~Hz}$. Our analysis of the feasibility of this setup indicates that branching fractions as small as $10^{-12}-10^{-14}$ can be probed. This experimental protocol benefits from the fact that a search for invisible modes is penalized for weak coupling only in the production of the new particle. If successfully implemented, this experiment is an exquisite probe of particles with mass below approximately $4 \mathrm{MeV}$ that lie in the poorly constrained supernova "trapping window" that exists between $100 \mathrm{keV}$ and $30 \mathrm{MeV}$. Such particles have been invoked as mediators between dark matter and nucleons, explain the proton radius and $(g-2)_{\mu}$ anomalies, and potentially power the shock wave in type II supernovae. The hadronic axion could also be probed with modifications to the proposed setup.
\end{abstract}

DOI: 10.1103/PhysRevD.99.035025

\section{INTRODUCTION}

Weakly coupled particles with mass below the megaelectron-volt scale arise in a number of extensions of the Standard Model. They are natural ingredients of the dark sector-either as dark matter candidates or as mediators to the dark matter [1]. They may also play a role in solving the gauge hierarchy and vacuum energy problems through cosmological evolution and could conceivably be responsible for powering the shock wave necessary to trigger type II supernovae $[2,3]$. High energy colliders do not have the statistical sensitivity to search for these particles-while they operate well above the energy threshold necessary to produce these particles, colliders do not have the luminosity to overcome the small coupling of these particles. In this paper, we propose a new method for a high statistics search for such particles.

We aim to search for invisible particles in nuclear gamma decays using the following protocol. We place a radioactive source in the middle of a hermetically sealed detector.

\footnotetext{
*hramani@berkeley.edu
}

Published by the American Physical Society under the terms of the Creative Commons Attribution 4.0 International license. Further distribution of this work must maintain attribution to the author(s) and the published article's title, journal citation, and DOI. Funded by SCOAP .
The source is chosen so that its decay triggers a gamma cascade in the daughter nucleus. The photons in the cascade are accurately counted, and their energies are measured. An invisible decay mode would manifest itself as a missing photon in this count. As a concrete example, consider ${ }^{60} \mathrm{Co}$, which undergoes a $\beta$ decay to ${ }^{60} \mathrm{Ni}$ (see Fig. 2). This decay (with high probability) places ${ }^{60} \mathrm{Ni}$ at a $2.51 \mathrm{MeV}$ excited state, which deexcites into another excited state by emitting a 1.17 MeV gamma. This excited state subsequently decays to the ground state by emitting a $1.33 \mathrm{MeV}$ gamma. A decay in which a $1.17 \mathrm{MeV}$ gamma is not followed by a $1.33 \mathrm{MeV}$ gamma would indicate the presence of a new light, weakly coupled particle. The ready availability of the ${ }^{60} \mathrm{Co}$ source allows a high statistics search, potentially enabling a probe of weakly coupled particles. A major advantage of this scheme is that the search for an invisible decay only suffers the cost of the weak coupling in the production of the particle, unlike other proposed schemes that rely on production and subsequent decay/scattering of the light particle. While there are experiments such as NA64 [4], LDMX [5], and BDX [6] that directly probe missing momentum/energy produced due to new particles coupled to electrons, there have not been similar efforts to probe baryonic interactions. Hunting for new forces in nuclear decays has been considered in early Higgs searches and axion searches [7-25], in which visible decays were nonetheless required. There has been a recent 
revival of similar ideas, albeit with detecting the final state involving an electron coupling in Refs. [26-28]. There have been few attempts at looking for the QCD axion in invisible nuclear decays in Refs. [29-31], albeit at lower energies and statistics.

In this paper, we analyze the experimental strategies necessary to implement the above scheme in a high statistics environment. First, it is essential that the experiment observes all the photons produced in the decay. We aim to accomplish this using a sufficiently large scintillation detector so that there are enough radiation lengths in the detector to contain all the decays. Second, the experiment needs to avoid pileup of events while maintaining high statistics. We aim to accomplish this goal by using plastic scintillators that have short (approximately $10 \mathrm{~ns}$ ) decay times and employing modular construction to separate events spatially. Finally, the scheme would have to distinguish the signal from a variety of systematic backgrounds such as misidentification of the gammas, potential confusion introduced by soft-Compton scattering, and population of other nuclear levels by the decaying source.

The feasibility of such an experiment is the focus of this paper. Since the missing particles are produced in the decays of nuclear isomers, the experiment is maximally sensitive to particles coupled to baryons. Our results indicate that this scheme has the potential to probe invisible branching fractions $\sim 10^{-12}-10^{-14}$. This is of significant interest; there are poor limits on particles with mass $\gtrsim 100 \mathrm{keV}$ that couple to baryons [1]. Several dark matter experiments are presently under development to search for interactions between the dark matter and the Standard Model mediated by particles around this mass scale. Particles in this parameter space have also been invoked to explain the proton radius and $(g-2)_{\mu}$ anomalies. Moreover, such light particles can be produced in type II supernovae, and their cosmic populations can potentially be discovered in current dark matter detectors. A light, weakly coupled particle in this scale could significantly affect the dynamics of type II supernovae, potentially resolving long-standing puzzles associated with the production of shock waves necessary to trigger such explosions. An experiment that can probe invisible branching fractions $\sim 10^{-12}-10^{-14}$ thus has significant phenomenological implications.

We start by presenting a simple toy model in Sec. II, in which we review current bounds and identify the experimentally accessible parameter space. Following this, we describe the experiment in greater detail in Sec. III. Section IV deals with the event selection. Sensitivity and backgrounds are treated in Sec. V, while the technological challenges and further improvements are presented in Sec. VI. Finally, we conclude in Sec. VII.

\section{TOY MODEL}

Consider the operator

$$
\mathcal{L}=g_{p} \phi \bar{p} p,
$$

describing the interactions of a light scalar $\phi$ with protons $p$. Such a scalar is a popular way to couple nucleons and dark matter. $\phi$ can be emitted during nuclear decays and can be probed by this proposed experiment. Taking this as our benchmark model, we quote the sensitivity of our experiment in terms of the coupling $g_{p}$.

There is a variety of constraints on $\phi$. Major constraints arise from astrophysics; cooling of stars/supernovae from energy carried away by this scalar. These are treated in detail in various texts [32], and we summarize the major results here. There are strong constraints on $g_{p}$ from horizontal branch stars and red giants when the mass of $\phi$ is less than $100 \mathrm{keV}$. Above $100 \mathrm{keV}$, there are limits from the cooling of SN1987A up to $100 \mathrm{MeV}$. However, for moderately large couplings, $\phi$ is trapped in the supernova and does not contribute to cooling. This trapping window is a prime target for the proposed experiment.

In addition to these astrophysical constraints, there are direct constraints from terrestrial experiments on coupling to nucleons. These are summarized in Ref. [33] and are relatively weak. Additional constraints can be placed on this scenario from UV completing this model. These are somewhat model dependent. For example, this nucleon coupling can be generated via heavy quark couplings or through gluons (via the operator $\phi G G$ ). Limits from kaon decays can set limits on these models. These were considered in detail in Ref. [34]. Here, we instead consider coupling to just the strange quark, which is not as constrained.

Starting with $\mathcal{L} \supset y_{q} \phi \bar{q} q$, where $\mathrm{q}$ is any light quark, leads to both meson coupling as well as proton (nucleon) coupling,

$$
\mathcal{L} \supset \frac{y_{q}}{m_{q}} \phi\left(m_{\pi}^{2} \pi^{+} \pi^{-}+f_{p}^{q} m_{p} \bar{p} p\right),
$$

where the $f_{p}^{q}$ for light quarks is tabulated in Ref. [35]. Here, $g_{p}=\frac{g_{q}}{m_{q}} f_{p}^{q} m_{p}$. The former term in the effective Lagrangian results in a new decay channel $K^{+} \rightarrow \pi^{+} \phi$. The branching fraction in the $m_{K} \gg m_{\pi} \gg m_{\phi}$ limit is given by

$$
\frac{\Gamma_{K \rightarrow \pi \phi}}{\Gamma_{K \rightarrow \mu \nu_{\mu}}}=\frac{3}{4}\left(\frac{g_{p} f_{\pi} m_{\pi}^{2}}{m_{K} m_{\mu} m_{p} f_{p}^{q}}\right)^{2} .
$$

The branching ratio for invisible decays of charged kaons is constrained to $\operatorname{Br}(K \rightarrow \pi \phi) \leq 1.7 \times 10^{-10}$. Since $f_{p}^{q}$ is largest for strange quarks, scalars coupled to just strange quarks then sets the most pessimistic limit $g_{p} \leq 4.10^{-5}$.

\section{A. Reach}

Our analysis of the experimental reach suggests a sensitivity to invisible decay modes with a branching ratio of approximately $10^{-12}-10^{-14}$. In this subsection, we describe the conversion between this experimental sensitivity and the coupling $g_{p}$. 


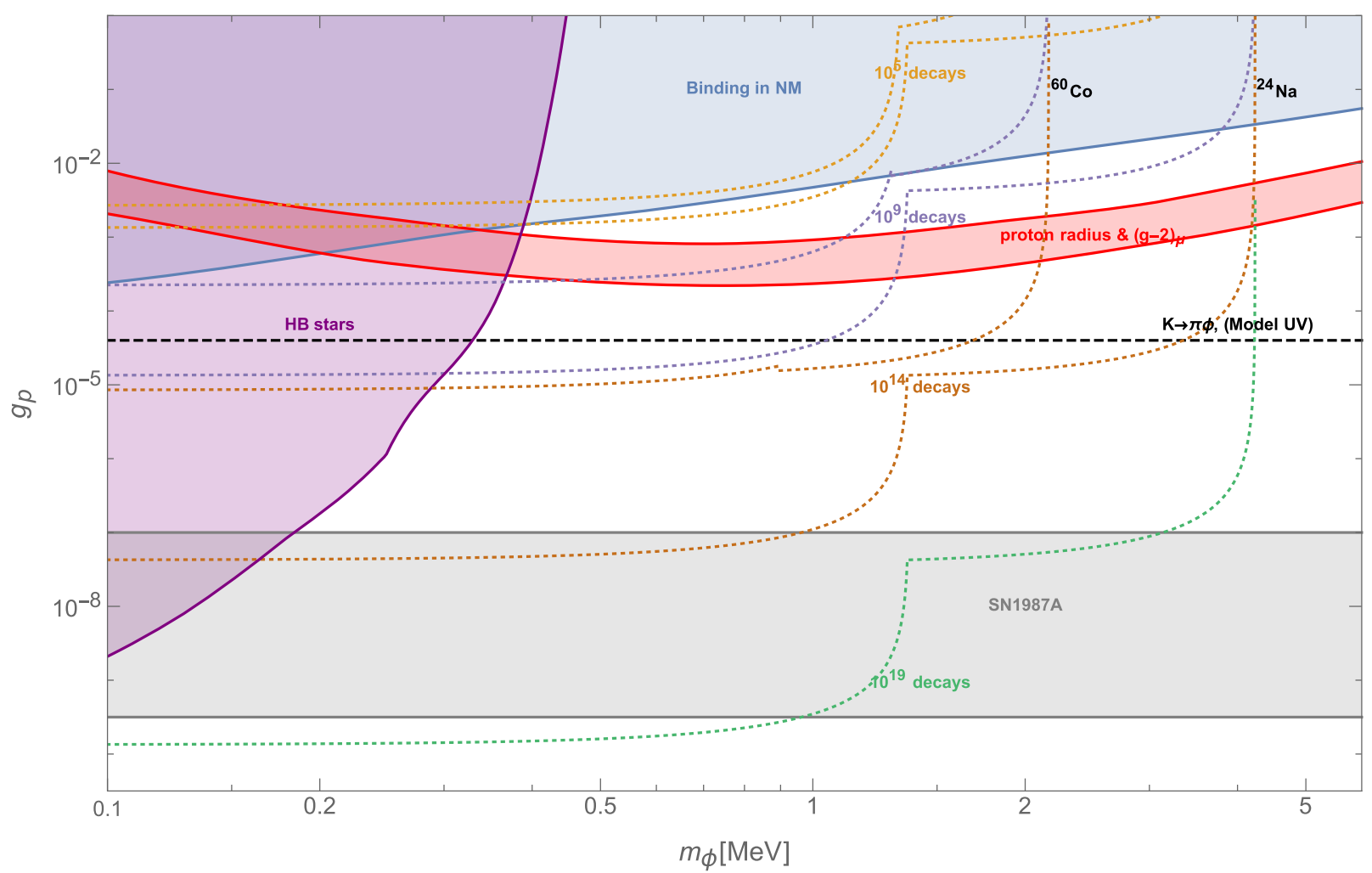

FIG. 1. Reach for ${ }^{60} \mathrm{Co}$ and ${ }^{24} \mathrm{Na}$ experimental proposals for $10^{5}, 10^{10}, 10^{14}$, and $10^{19}$ decaying mother nuclei. Also shown are direct limits from binding in nuclear matter [33], limits from cooling in SN1987A and horizontal branch stars [32] and indirect limits from meson decays in a UV complete model (refer to the text). Also shown in red is the region that could explain the proton radius and muon g-2 puzzles simultaneously [33].

This particular computation strictly applies to couplings to protons and ignores the coupling to neutrons because of the ease of porting known photon matrix elements to those of $\phi$. The estimate of the branching fraction also requires knowledge of the specific nuclear transitions. In our proposed experiment, we consider two promising radioactive sources (see Sec. III for details): ${ }^{60} \mathrm{Co}$ and ${ }^{24} \mathrm{Na}$. In both these cases, the relevant gamma transitions are $E_{2}$ transitions.

For an $E_{2}$ transition, the quadrupole Hamiltonian that is induced by this Yukawa coupling is (see for example Ref. [27])

$$
H_{\text {int }}^{\phi}=g_{p} R_{p}^{i} R_{p}^{j} \nabla_{i} \nabla_{j} \phi(k),
$$

where $\phi(k)$ is the free-particle wave function. Comparing this to

$$
H_{\mathrm{int}}^{\gamma}=e R_{p}^{i} R_{p}^{j} \nabla_{i} \epsilon_{j}
$$

notice that for a massive scalar the momentum $k=$ $\sqrt{\omega^{2}-m_{\phi}^{2}}$, where $\omega$ is the energy gap of the transition. Putting this all together,

$$
\frac{\Gamma(\phi)}{\Gamma_{\gamma, E_{2}}} \sim \frac{1}{2}\left(\frac{g_{p}}{e}\right)^{2}\left(1-\frac{m_{\phi}^{2}}{\omega^{2}}\right)^{\frac{5}{2}} .
$$

Assuming a $100 \%$ efficiency in photon detection (which is realized in ${ }^{24} \mathrm{Na}$ as explained later), we plot the reach for $10^{5}, 10^{10}$, and $10^{14}$ decaying mother nuclei. These roughly correspond to $10^{-2}, 10^{3}$, and $10^{7} \mathrm{~Hz}$ triggering frequency for a one-year run. The reach is plotted in Fig. 1 for experiments using ${ }^{60} \mathrm{Co}$ and ${ }^{24} \mathrm{Na}$. If this sensitivity is successfully attained, this experiment will probe the entire trapping window of mediators in the mass range $100 \mathrm{keV}$ to approximately $1.3 \mathrm{MeV}$.

Also plotted in Fig. 1 in red is the parameter space for a hypothetical scalar, which would explain the proton radius puzzle [33] as well as the muon g-2 anomaly. While only the product of proton and muon couplings is fixed by the proton radius puzzle requirement, the proton coupling itself is uniquely fixed by the muon coupling required to solve the muon g-2 anomaly [36]. This experimental proposal will be able to probe this parameter space up to $4 \mathrm{MeV}$ mass.

\section{EXPERIMENTAL CONCEPT}

From Eq. (6), it follows that an experiment searching for the disappearance of a gamma ray with energy $E_{\gamma}$ is sensitive to a scalar $\phi$ with a mass up to $E_{\gamma}$. A positive signal would allow the measurement of the coupling term $g_{p}$ but not of $m_{\phi}$. The experimental sensitivity goes as the 
ratio between the decay rate into the dark sector and the standard one:

$$
\hat{g_{p}}=\frac{g_{p}}{e} \propto \sqrt{\frac{\Gamma(\phi)}{\Gamma_{\gamma}}} .
$$

Figure 1 shows two unexplored regions, corresponding to $\left[10^{5}, 10^{14}\right]$ decays and to $=10^{19}$ decays. While the latter is hardly feasible with current detector technology, it is possible to probe the first region with one year of live time, provided that the experimental apparatus can sustain a count rate of approximately $10 \mathrm{MHz}$ and has containment efficiency $\varepsilon_{c} \geq\left(1-10^{-14}\right)$. Such a high efficiency is only reachable with a liquid or solid detector with a large enough continuous active volume.

Additional requirements are imposed by the choice of the gamma emitting isotope. To maximize the accessible parameter space region, we want $E_{\gamma}$ to be large, above the current horizontal branch stars exclusion limits at approximately $200 \mathrm{keV}$. Moreover, the considered decay must offer a clean signature with no intrinsic backgrounds. Restricting our discussion to $\alpha$ and $\beta$ decaying isotopes only, we need to be able to detect distinct energy depositions for the $\alpha / \beta$ and the daughter gamma ray(s). In practice, any radioactive source has a finite size, and $\alpha$ particles of few $\mathrm{MeV}$ have a range of order of tens of nanometers in average- $Z$ materials. This strongly suppresses the $\alpha$ detection efficiency for all those atoms which are not on the surface of the source and leads us to the choice of $\beta$ decaying nuclei. One possibility is to select an isotope that undergoes $\beta$ decay followed by a single gamma deexcitation of the daughter nucleus, such as ${ }^{137} \mathrm{Cs}$. The signal signature would be an energy deposition compatible with that of the $\beta$ in a location next to that of the source. A much more identifiable signature would be that of a $\beta$ decay followed by two gammas in cascade, as for example in the case of ${ }^{60} \mathrm{Co}$. A signal-like event would be characterized by the $\beta$ energy deposition in the vicinity of the source and a gamma energy deposition elsewhere in the detector volume. The distinction between the two energy depositions requires a specific space resolution, which depends on the energies of the involved particles. Typically, gamma from nuclear deexcitations are emitted within picosecond or nanoseconds from the original decay and would be considered in coincidence with it for most detector technologies. The requirement of a double coincidence within a $\mathcal{O}($ ns) time window strongly suppresses random coincidences and background events induced by isotopes decaying in cascade. On the other hand, the potential presence of intrinsic backgrounds induced by the source itself and mimicking the gamma disappearance must be considered in the isotope choice.

The practicality of the source production and usage imposes additional requirements on the selection of the

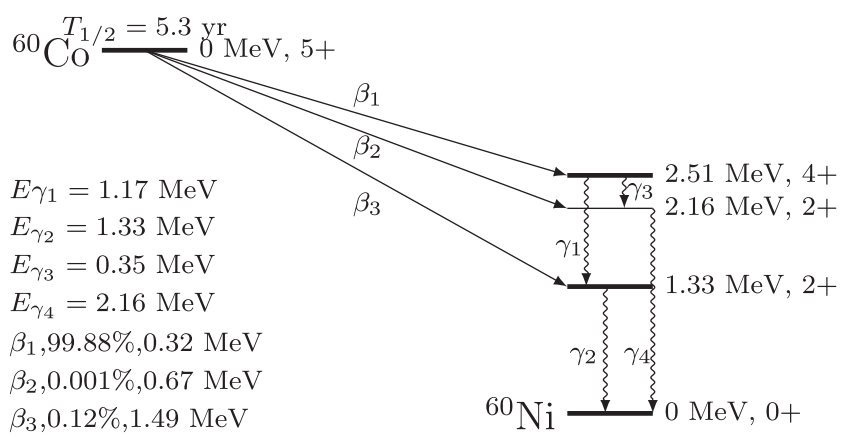

FIG. 2. Decay scheme of ${ }^{60} \mathrm{Co}$.

isotope and of the detector technology. First of all, the isotope half-life has to be long enough to allow the source transportation to the experiment site, its insertion in the detector, and a measurement time sufficient to collect the required statistics. Thus, isotopes with a half-life $\gtrsim 1 \mathrm{yr}$ are preferable. Alternatively, we could envisage the repeated production and insertion of the source in the experimental apparatus, provided that this is close enough to the production site. Such a choice allows the use of isotopes with half-lives down to several hours, but imposes the capability to insert and extract the source in the detector without affecting its performance, and the availability of a long-term dedicated source production facility, e.g., a beam line. Furthermore, the necessity of measuring the $\beta$ in a given location restricts our choice to solid state sources on thin enough materials to minimize the self-absorption. Finally, isotopes for which a production technology exists with industrial standards are preferable.

Two isotopes that fulfill most of these criteria are ${ }^{60} \mathrm{Co}$ and ${ }^{24} \mathrm{Na}$. With ${ }^{60} \mathrm{Co}$ (Fig. 2), we can search for the disappearance of the $1.33 \mathrm{MeV}$ gamma. The signal signature is therefore a twofold energy deposition by the $\beta$ and the $1.17 \mathrm{MeV}$ gamma. On the one hand, ${ }^{60} \mathrm{Co}$ is a commercially available isotope with a half-life that perfectly fits the live time of a hypothetical experiment. On the other hand, the relatively low end point of the $\beta$ spectrum $(0.32 \mathrm{MeV})$ and the small difference between the energy of the two gammas impose strict requirements in terms of energy threshold and resolution. Furthermore, ${ }^{60} \mathrm{Co}$ is affected by an intrinsic background in that it has a $0.12 \%$ branching ratio into the $1.33 \mathrm{MeV}$ excited state of ${ }^{60} \mathrm{Ni}$. A signal-like event can be detected if most of the energy is carried away by the antineutrino and if the $1.33 \mathrm{MeV}$ gamma undergoes a soft-Compton scattering in proximity of the source and is then fully absorbed elsewhere. The actual importance of this background strongly depends on the detector material and on the spatial resolution.

Scientifically, the perspectives offered by ${ }^{24} \mathrm{Na}$ are more promising. The signal in this case would be given by the detection of the $\beta$ with the largest branching ratio $\left(\beta_{2}\right.$ in Fig. 3) followed by $\gamma_{5}$ with $2.75 \mathrm{MeV}$, with the 


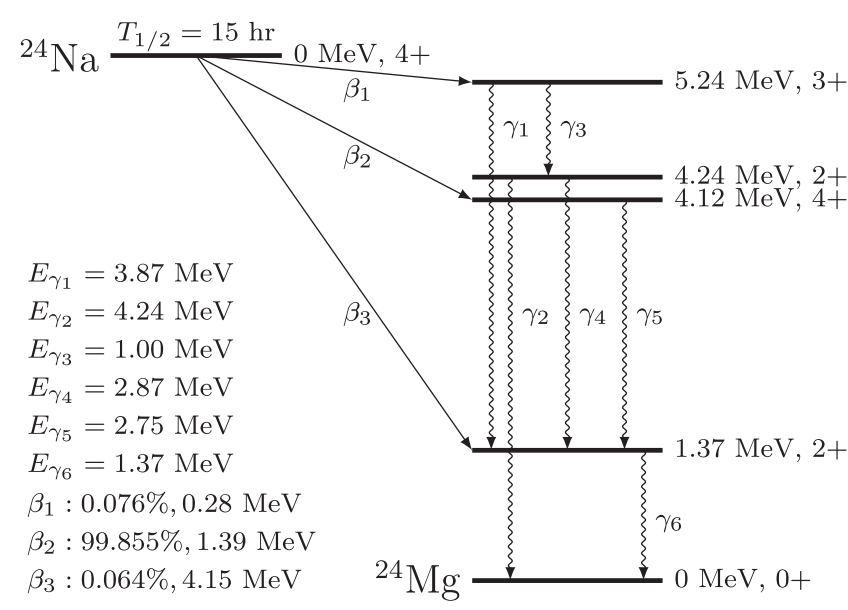

FIG. 3. Decay scheme of ${ }^{60} \mathrm{Na}$.

corresponding disappearance of $\gamma_{6}$ with $1.37 \mathrm{MeV}$. For ${ }^{24} \mathrm{Na}$, there are two intrinsic backgrounds, both of which can be easily suppressed. As shown in Fig. 3, the parent nucleus can decay via the emission of $\beta_{3}$. If $\beta_{3}$ has enough energy and $\gamma_{6}$ is absorbed in vicinity of the source, this could be misinterpreted as the sum of $\beta_{2}$ and $\gamma_{5}$. This type of event can easily be removed with the requirement that $\gamma_{5}$ has to be detected sufficiently far from the source. A second background arises in the main branch if $\beta_{2}$ has very small energy and $\gamma_{6}$ is absorbed next to the source. This possibility can be rejected by setting an upper limit smaller than $E_{\gamma_{6}}$ for the energy deposition next to the source. The main drawback of ${ }^{24} \mathrm{Na}$ is its short half-life of about $15 \mathrm{hr}$, which necessitates the placement of the detector in the vicinity of the source production site as well as the use of a detector technology which allows the repeated source insertion and removal. In the long term, one could envisage a two-stage approach in which ${ }^{60} \mathrm{Co}$ is first used to test and improve the technology, followed by a ${ }^{24} \mathrm{Na}$ phase with more ambitious physics goals.

Finally, a small fraction of the decays proceeds through cascades with second photons with much higher energy than the benchmark photons we discuss for both ${ }^{60} \mathrm{Co}$ and ${ }^{24} \mathrm{Na}\left(\gamma_{3}[\right.$ trigger $]+\gamma_{4}[$ miss $\left.]\right)$. This increases the reach to higher $\phi$ masses, albeit with lesser sensitivity. This shows up as a kink in Fig. 1.

\section{A. Design characteristics}

The required high containment efficiency and time resolution can only be achieved with liquid or solid scintillators. If a ${ }^{24} \mathrm{Na}$ source is to be used, a promising design is that of a stack of solid scintillator modules with the source as a thin foil at the center, as depicted in Fig. 4. To minimize the dead volume, one can substitute the standard reflective foils with ultrathin nanofabricated coatings, for which the technology is readily available. The detection efficiency can be maximized by coupling light

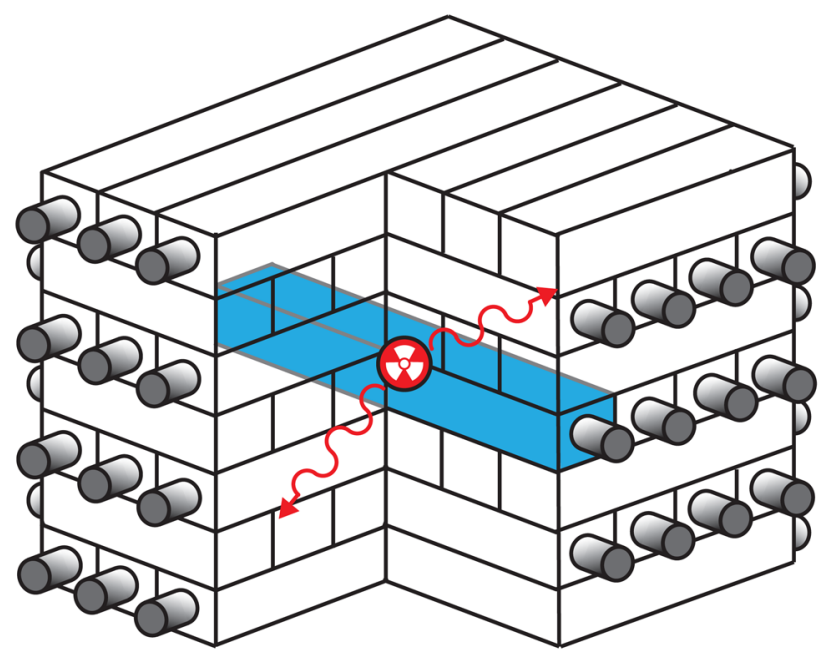

FIG. 4. Schematic rendering of the experimental design: the scintillator modules are stacked in layers with alternating orientations and are coupled to light detectors (grey cylinders) on both ends. The central module, used for triggering on $\beta$ events, is shown in blue and can be made of a different material or size.

detectors (e.g., photo multiplier tubes or silicon photo multipliers) at the two ends of each module. While some scintillating crystals offer higher light yields (LYs) of up to $6 \times 10^{4}$ photons/MeV [37], their maximum size is limited by crystal growth technology, and their cost tends to scale up quickly with size. Plastic scintillators, on the other hand, can typically sustain a higher count rate thanks to lower decay times, are cheaper, and can be molded in almost arbitrary shape and size. Their main drawback is the light output, limited to approximately $10^{4}$ photons/MeV [37].

We developed a full GEANT4 [38] Monte Carlo (MC) simulation to evaluate the containment efficiency as a function of the active detector size and quantify the importance of the intrinsic ${ }^{60} \mathrm{Co}$ background described above. For simplicity, we simulated a cubic active volume of side $l$, composed of rectangular cuboids of volume $l \times d \times d$ arranged in alternating orientations.

In the following calculations, we assume a LY of $10^{4}$ photons $/ \mathrm{MeV}$ (typical for a plastic scintillator such as BC-404 [37]) and conservatively scale it down by a factor of 3 to account for the self-adsorption and possible inefficiencies in the light propagation to the detector, a $25 \%$ quantum efficiency (QE) for the light detectors, and an energy resolution given by

$$
\sigma_{E}=\frac{\sqrt{3 \cdot L Y \cdot Q E \cdot E}}{L Y \cdot Q E} .
$$

\section{EVENT SELECTION}

As mentioned above, the signal signature is an energy deposition compatible with that of the $\beta_{1}$ in the module(s) next to the source and an energy compatible with that of 
$\gamma_{1}$ for ${ }^{60} \mathrm{Co}$ deposited elsewhere, or with $\beta_{2}$ and $\gamma_{5}$ for ${ }^{24} \mathrm{Na}$. In order to mitigate backgrounds, we segment the detector into three regions (see Fig. 4). First, we have a central module around the source of which the purpose is to measure the beta from the source. This module will size approximately a $\sim \mathrm{cm}$ in side, so it can completely stop the approximately mega-electron-volt betas produced by the source. Surrounding this central module, there will be an inner module of thickness of approximately $10 \mathrm{~cm}$, corresponding to one radiation length of the expected gammas. The inner modules are surrounded by outer modules that extend to sufficiently many radiation lengths to achieve the necessary containment.

Our event selection protocol works as follows: we demand that there is an energy deposition in the central module consistent with the initial beta. We then demand that the subsequent gammas deposit all of their energy in the inner modules of the detector. If there is any energy deposited in the outer modules within the approximately nanosecond timing resolution of the experiment or if the gamma ray energy deposited in the inner module is inconsistent with the expected energy, we veto the event.

This strategy sacrifices $\mathcal{O}(1)$ of the signal, where the gammas travel a few radiation lengths before scattering or have soft collisions in these inner modules. On the other hand, this eliminates the need to carefully reconstruct activity that occurs in the outer modules which house most of the volume of the detector.

\section{SENSITIVITY AND BACKGROUNDS}

To estimate the efficacy of the above signature, we define a region of interest (ROI) $\left[E_{\gamma}-n \sigma_{E}, E_{\gamma}+n \sigma_{E}\right]$ around the energy of the considered gamma. All processes which can mimic this signature represent possible backgrounds which can hinder a discovery.

To quantify the effect of each background, we compute the $3 \sigma$ discovery sensitivity as a function of the live time of the measurement $t$ and of the other experimental parameters. In general, the number of signal events $s$ can be written as

$$
s=\varepsilon_{t} \cdot \varepsilon_{\mathrm{ROI}} \frac{\Gamma(\phi)}{\Gamma_{\gamma}} \cdot \varepsilon_{M C} \cdot A \cdot t,
$$

where $\varepsilon_{t}$ is the trigger efficiency, $\varepsilon_{\mathrm{ROI}}$ is the fraction of signal events with energy deposition in the inner modules falling in the selected ROI, $\varepsilon_{M C}$ is the containment efficiency, and $A$ is the source activity. The number of background events is given by

$$
b=\varepsilon_{t} \cdot \varepsilon_{b} \cdot A \cdot t,
$$

where $\varepsilon_{b}$ is the probability of a specific background to induce an event in the ROI. In all calculations, we can safely assume $\varepsilon_{t}=\varepsilon_{M C}=1$ and compute the sensitivity as a function of the exposure $A \cdot t$. We define the discovery sensitivity as that value of $\Gamma(\phi) / \Gamma_{\gamma}$ for which an experiment has a $50 \%$ probability to measure a positive signal above background with a significance of at least $3 \sigma$. We compute this following the heuristic counting approach described in Ref. [39].

\section{A. Photon miss}

The first background arises if the gamma under investigation is not absorbed in the active detector volume. In our design, this can happen only in the source itself or if the gamma escapes undetected. Therefore, the source must consist of a thin enough foil to make the self-adsorption negligible, and the detector size must be such that $\varepsilon_{M C} \geq\left(1-10^{-A \cdot t}\right)$. In order to reach a $\hat{g}_{p} \sim 10^{-7}$, the total detector size must cover 32 interaction lengths, corresponding to approximately $10 \mathrm{~m}$ for BC-404. Such a high containment represents a major technological challenge for the proposed design, as the presence of empty and dead volumes has to be avoided at any cost. Empty volumes can presumably be avoided using scintillator modules with nontrivial shapes to avoid direct lines of sight between the source and the outside world, together with the aforementioned thin film reflective coatings.

\section{B. 1.33 MeV gamma mimicking 1.17 MeV gamma}

In the ${ }^{60} \mathrm{Co}$ case, another background is induced by the misreading of a $1.33 \mathrm{MeV}$ gamma as a $1.17 \mathrm{MeV}$ one. This is possible if the energy resolution is such that a nonnegligible fraction of $1.33 \mathrm{MeV}$ events can fall in the ROI. It can only occur if the decay follows the weaker ${ }^{60} \mathrm{Co}$ branch and if $E_{\beta_{2}}<0.32 \mathrm{MeV}$. The background efficiency is

$\varepsilon_{b}=0.0012 \cdot P\left(E_{\beta_{2}}<0.32 \mathrm{MeV}\right) \cdot P\left(E_{\gamma_{2}} \in \mathrm{ROI}\right)$.

MC simulations give $P\left(E_{\beta_{2}}<0.32 \mathrm{MeV}\right) \simeq 0.8$, with a weak dependence on the scintillator module size, $d$. Using this value, we obtain the discovery sensitivity as a function of the exposure shown in Fig. 5.

\section{Soft-Compton events}

For ${ }^{60} \mathrm{Co}$, the weak decay branch causes a second type of background, if $E_{\beta_{2}}<0.32 \mathrm{MeV}$ and if $\gamma_{2}$ makes a softCompton scattering in the same scintillator module(s) where $\beta_{2}$ is detected. The background efficiency is

$$
\begin{aligned}
\varepsilon_{b}= & 0.0012 \cdot P(E(\text { central })<0.32 \mathrm{MeV}) \\
& \cdot P(E(\text { elsewhere }) \in \mathrm{ROI}) .
\end{aligned}
$$

The probability for these events to happen increases with the size of the scintillator modules, as shown in Fig. 6. With $l \lesssim 1 \mathrm{~cm}$, this background is small enough to allow 


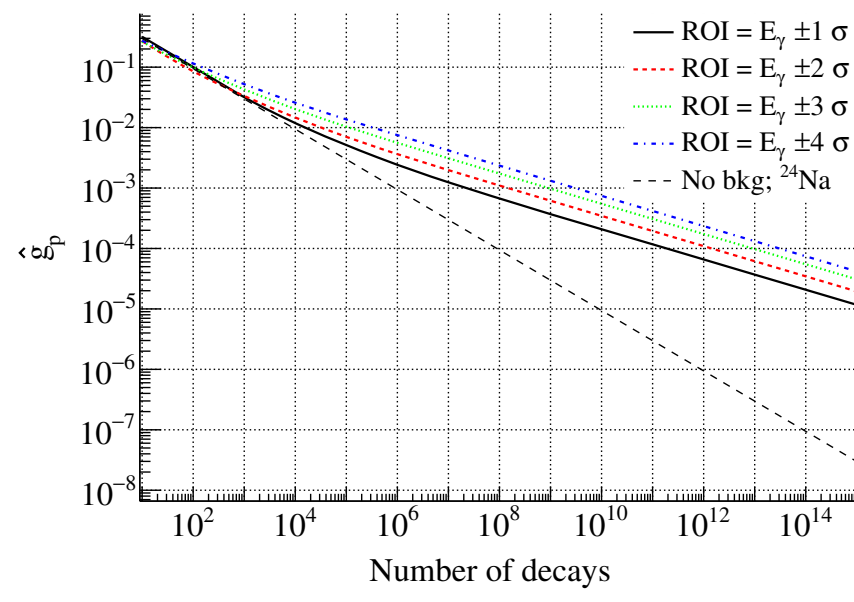

FIG. 5. Discovery sensitivity curve at $3 \sigma$ significance with ${ }^{60} \mathrm{Co}$ for different choices of the ROI. The dashed line shows the case with no background or with ${ }^{24} \mathrm{Na}$.

reaching a sensitivity of $\hat{g}_{p} \sim 10^{-5}$ with one year of live time. In practice, though this is not illustrated in the cartoon schematic in Fig. 4 nor tested in our simulation, we envisage the use of smaller detector modules in vicinity of the source and of larger modules in the outer region. Due to this intrinsic background, ${ }^{60} \mathrm{Co}$ allows us to cover only about half of the parameter space composed by the nuclear matter and the SN1978A excluded regions (Fig. 1). Advanced event reconstruction algorithms might help us identify softCompton events, but it is not possible to estimate their efficiency without a dedicated study, which is out of the scope of this work.

\section{Radioactive contaminants}

Radioactive contaminants in the detector can be a source of background. For these contaminants to matter, they would have to deposit energy in the central module that is

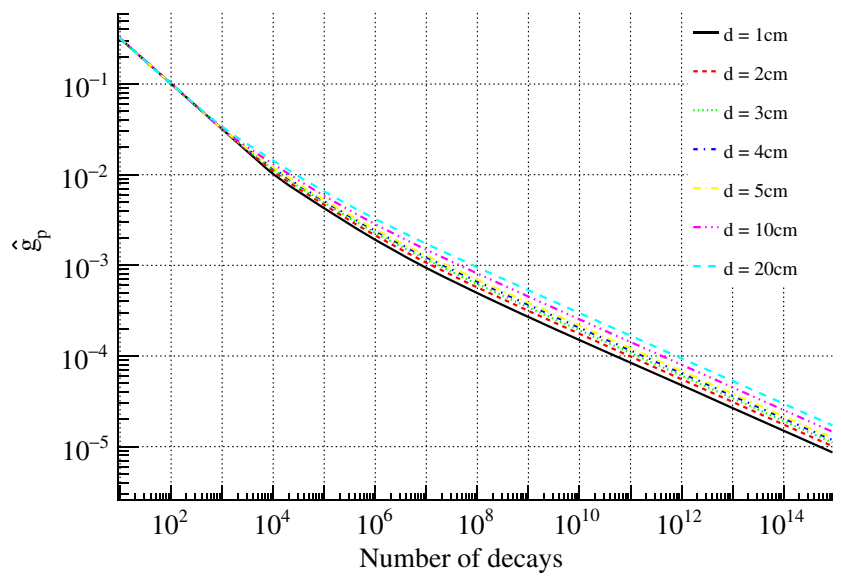

FIG. 6. Discovery sensitivity curve at $3 \sigma$ significance with ${ }^{60} \mathrm{Co}$ with the inclusion of the soft Compton for different sizes of the scintillator module. consistent with the initial beta and lead to energy deposition in the inner modules that is consistent with the single gamma expected in the decay chain. A list of common contaminants and their expected activity in scintillators can be found in Ref. [40]. The most dangerous contaminant for ${ }^{60} \mathrm{Co}$ is ${ }^{40} \mathrm{~K}$, which decays (with a $10 \%$ branching fraction) to ${ }^{40} \mathrm{Ar}$ via electron capture, emitting a $1.46 \mathrm{MeV}$ gamma. This contaminant is estimated to occur with an activity $\sim$ milli becquerel in the approximately kilogram volume of the inner modules [40]. If this gamma undergoes a soft scattering in the central module and subsequently rescatters in the inner module, it could mimic our signal in ${ }^{60} \mathrm{Co}$. With branching fraction and phase space suppression, this yields worrisome events at a rate of approximately $10^{-5} \mathrm{~s}^{-1}$. If the ${ }^{60} \mathrm{Co}$ experiment is operated at an event rate of approximately $10 \mathrm{MHz}$, branching fractions approximately $10^{-12}$ can be probed before this background becomes a problem. This background is then comparable to other intrinsic backgrounds in ${ }^{60} \mathrm{Co}$. It is possible to further mitigate this background through a minor sacrifice in the signal. The signal from ${ }^{60} \mathrm{Co}$ involves a $0.32 \mathrm{MeV}$ beta followed by a $1.17 \mathrm{MeV}$ gamma. By requiring that the beta from the ${ }^{60} \mathrm{Co}$ decay has less energy than $0.2 \mathrm{MeV}$, the total energy in the ${ }^{60} \mathrm{Co}$ decay is resolvably different from the energy produced in ${ }^{40} \mathrm{~K}$, suppressing this background. There do not appear to be comparably worrisome contaminants for ${ }^{24} \mathrm{Na}$.

\section{E. Cosmics and neutrons}

The requirement that the event only contains energy deposition in the inner and central modules is a powerful way to discriminate against backgrounds induced by cosmic rays. With the exception of neutrons, cosmic ray activity will lead to a trail of energy deposition in the detector and can be vetoed. Environmental neutrons can potentially cause backgrounds if they get captured in a nucleus, leading to deexcitation through gammas that could be in the right energy range. However, the inner and central modules of the detector are at least approximately 30 neutron radiation lengths (approximately $10 \mathrm{~cm}$ ) away from the environment. We thus expect the inner regions to be self-shielded from environmental neutrons. Neutrinos are the only environmental source that can penetrate into the inner modules of the detector. They could be a cause for concern if they are inelastically absorbed in nuclei, leading to deexcitation through gamma rays. The expected rate for such events in the inner modules of the detector is approximately $10^{-2} / \mathrm{yr}$ and is thus not a concern.

\section{F. Dead regions}

Solid state scintillators can have dead layers, where energy deposition leads to highly suppressed light emission. These dead layers can be caused due to oxidation, humidity, and mechanical damage (see Ref. [41] and references therein). In plastic scintillators, the typical thickness of these 
dead layers is approximately $1-10 \mu \mathrm{m}$. [41]. In the outer modules of the detector, the sole purpose of which is to identify the existence of a second photon in the event (rather than a precise reconstruction of the energy itself), a dead region would be problematic only if all of the photon's energy were absorbed in that region. However, upon absorption, a mega-electron-volt gamma would produce a mega-electron-volt electron, which would travel a distance $\sim \mathrm{cm}$ before being stopped. Since this is much bigger than the expected thickness of the dead region, we do not expect a background from their existence in the outer modules.

In the inner module, where energy reconstruction is important, a dead region can be problematic if it causes misidentification of energy. For example, if ${ }^{60} \mathrm{Co}$ decays through $\beta_{2}$ or ${ }^{24} \mathrm{Na}$ decays through $\beta_{3}$ and if the energy of the outgoing photon is misidentified due to the photon going through a dead region, it is possible for these regions to cause backgrounds. In ${ }^{24} \mathrm{Na}$, where the two gammas are well separated in energy, this should not be a problem. In ${ }^{60} \mathrm{Co}$, the gamma would have to lose approximately $100 \mathrm{keV}$ of energy in a dead region in order to get confused with the signal photon $\gamma_{1}$. However, the approximately $100 \mathrm{keV}$ electron produced in this absorption would travel a distance approximately $100 \mathrm{~mm}$ before stopping. Since the typical lengths of the dead layers are approximately $1-10 \mathrm{~mm}$, it does not seem likely that these dead regions would cause a background even for ${ }^{60} \mathrm{Co}$. In the event of a positive signal, since the problem of the dead layers is confined to potentially big (approximately $100 \mathrm{~mm}$ ) regions in the inner modules, they could be examined to observe the size of such regions [42].

The radiation dose experienced by the central module at an event rate of $10 \mathrm{MHz}$ operating for a year is approximately $10^{4}$ grays, smaller than the dosage ( $\gtrsim 10^{5}$ grays) necessary to cause damage to the module.

\section{TECHNOLOGICAL CHALLENGES AND POSSIBLE IMPROVEMENTS}

The experimental apparatus we propose features several technical challenges, which we believe can potentially be overcome with a careful design of all its components. First, a nontrivial trigger logic is necessary; it must be capable of selecting in real time only signal-like events while handling the high (approximately $10 \mathrm{MHz}$ ) data rate. This trigger would also have to allow operation in "background" mode to store all detected events for a detailed characterization of the detector response. The design of this trigger is beyond the scope of this work. However, we point out that triggers that need to operate at this high frequency with comparable efficiency are necessary for the LDMX experiment currently being developed at SLAC [43]. Second, the required containment efficiency has to be achieved while avoiding the presence of significant dead volumes (see estimates above) and minimizing source thickness to prevent selfabsorption. Finally, in an experiment using ${ }^{24} \mathrm{Na}$, source production must be possible in the vicinity of the experimental apparatus. Given its high chemical reactivity, a

${ }^{23} \mathrm{Na}$ compound must be used, yielding the possibility of parasitic activation of other molecular components during neutron irradiation. A reasonable compound is one which either does not suffer from parasitic activation or that does not induce additional background and does not increase the total count rate.

Designs alternative to the one we proposed are also possible. For example, similar containment efficiencies are achievable with time projection chambers or large liquid scintillator experiments. The main disadvantages are limited spatial resolution and difficulties in repeated insertion and removal of the source. One concept worthy of further exploration might be to insert the inner module in a large liquid scintillator, which would serve as the outer module. This design would combine the rapid response necessary in the inner module to handle the high event rate while using the liquid scintillator to identify energy deposition that would trigger an event veto. Such a detector could potentially be installed in existing large liquid scintillator experiments. In addition, outer modules made out of a liquid scintillator would also mitigate concerns about dead regions in those modules.

In this work, we made use of a simplified geometry, of conservative assumptions for the parameters characterizing the scintillator and the light detectors, and of a trivial event selection in the analysis of the simulated data. A large space for improvement is available in both the hardware and the event reconstruction aspects. Light detectors with up to $35 \% \mathrm{QE}$ are available on the market, allowing for a strong improvement in energy resolution. The geometry and size of the scintillator modules can be optimized to maximize containment and minimize the intrinsic ${ }^{60} \mathrm{Co}$ backgrounds. Finally, event reconstruction algorithms using the information collected by the single modules can further improve the signal-to-background ratio.

From a strategic point of view, we propose a multistage approach. In a first phase, a ${ }^{60} \mathrm{Co}$ source can be used to develop, test, and prove the performance of the inner modules of the detector where energy reconstruction has to occur with high efficiency at a high rate. If successfully implemented, this phase can lead to a full scale ${ }^{60} \mathrm{Co}$ experiment that proves containment and background rejection of the outer modules. At a later stage, this setup can be operated with ${ }^{24} \mathrm{Na}$ at a facility with localized source production. Finally, a positive signal could be scrutinized with the use of additional sources inducing more complicated signatures (e.g., with multiple gammas), which can be hardly mimicked by external backgrounds.

\section{CONCLUSIONS}

Experiments in the intensity/statistics frontier have the ability to probe physics complementary to the high energy parameter space accessed by colliders. Given the existence 
of weakly coupled sectors such as those associated with the dark matter and dark energy, there are strong theoretical motivations to search for light, weakly coupled particles. While a successful implementation of this experiment covers the supernova "trapping" window, it is clear that there are significant challenges to probe parameters below the range constrained by supernova cooling bounds. Accessing this parameter space will make the experiment sensitive to a wider class of well-motivated particles with modifications (other nuclei) sensitive to even the QCD axion and axionlike particles in a range of parameters that are otherwise hard to constrain.

To probe this region, the experiment must be sensitive to branching fractions smaller than $10^{-19}$. This involves many obstacles that require additional technology development. Specifically, the required containment efficiency requires a larger detector size and techniques to handle source activity at the giga becquerel level. Event pileup can potentially be mitigated with an optimized choice of geometry wherein the detector modules are at some distance from the source. This would spread out the decays over multiple detection modules, preventing pileup. However, a complex trigger mechanism would be required to do simultaneous event reconstruction for multiple decays.

On the other hand, it is possible that a more optimized choice of nuclear states could alleviate the statistics necessary to probe these particles. For example, $M_{1}$ transitions in ${ }^{65} \mathrm{Ni}$ could be sensitive to the pseudoscalar axion. Furthermore, gamma cascades that involve forbidden photon transitions (e.g., $E_{0}$ or $M_{0}$ ) could offer significant advantages in the search for scalars, axions, and axionlike particles. Since these bosons do not carry spin, the branching fraction for axion/axionlike particle production in transitions between forbidden levels would be enhanced. If suitable levels are found, this will decrease the statistics needed to search for these particles as well as decrease the containment efficiency required.

\section{ACKNOWLEDGMENTS}

The authors sincerely thank M. Battaglieri, W. C. Haxton, Y. G. Kolomensky, and B. Schwingenheuer for providing very useful feedback and suggestions. S. R. was supported in part by the NSF under Grants No. PHY1638509 and No. PHY-1507160, the Alfred P. Sloan Foundation Grant No. FG-2016-6193, and the Simons Foundation Award Grant No. 378243. This work was supported in part by Heising-Simons Foundation Grant No. 2015-038. H. R. is supported in part by the U.S. Department of Energy (DOE) under Contract No. DEAC0205CH11231 and the BFS Fund, Grant No. 27660. G. B. and A. D. are supported by the DOE Office of Science under Contract No. DE-AC02$05 \mathrm{CH} 11231$ and by the DOE Office of Science, Office of Nuclear Physics, under Contract No. DE-FG0208ER41551. Some of this research was completed in Kavli institute of Theoretical Physics (KITP), supported in part by the National Science Foundation under Grant No. NSF PHY-1748958. Part of this work was also completed at Aspen Center for Physics, which is supported by National Science Foundation Grant No. PHY-1607611.
[1] D. Green and S. Rajendran, J. High Energy Phys. 10 (2017) 013.

[2] J. Hidaka and G. M. Fuller, Phys. Rev. D 76, 083516 (2007).

[3] W. DeRocco, P. W. Graham, D. Kasen, G. Marques-Tavares, and S. Rajendran, arXiv:1901.08596.

[4] D. Banerjee et al. (NA64 Collaboration), Phys. Rev. Lett. 118, 011802 (2017).

[5] J. Mans (LDMX Collaboration), EPJ Web Conf. 142, 01020 (2017).

[6] M. Battaglieri et al. (BDX Collaboration), arXiv:1406.3028.

[7] F. P. Calaprice, R. W. Dunford, R. T. Kouzes, M. Miller, A. Hallin, M. Schneider, and D. Schreiber, Phys. Rev. D 20, 2708 (1979).

[8] A. Zehnder, Phys. Lett. 104B, 494 (1981); 104, 494 (1981).

[9] J. F. Cavaignac et al., Phys. Lett. 121B, 193 (1983).

[10] G. D. Alekseev et al., JETP Lett. 36, 116 (1982).

[11] P. Lehmann, E. Lesquoy, A. Muller, and S. Zylberajch, Phys. Lett. 115B, 270 (1982); 115, 270 (1982).

[12] A. Zehnder, K. Gabathuler, and J. L. Vuilleumier, Phys. Lett. 110B, 419 (1982).
[13] V. D. Ananev et al., Yad. Fiz. 41, 912 (1985) [Sov. J. Nucl. Phys. 41, 585 (1985)].

[14] A. L. Hallin, F. P. Calaprice, R. W. Dunford, and A. B. Mcdonald, Phys. Rev. Lett. 57, 2105 (1986).

[15] F. T. Avignone III, C. Baktash, W. C. Barker, F. P. Calaprice, R. W. Dunford, W. C. Haxton, D. Kahana, R. T. Kouzes, H. S. Miley, and D. M. Moltz, Phys. Rev. D 37, 618 (1988).

[16] V. M. Datar, S. Fortier, S. Gales, E. Hourani, H. Langevin, J. M. Maison, and C. P. Massolo, Phys. Rev. C 37, 250 (1988).

[17] F. W. N. De Boer, J. Deutsch, J. Lehmann, R. Prieels, and J. Steyaert, J. Phys. G 14, L131 (1988).

[18] J. Dohner, J. Last, M. Arnold, S. J. Freedman, and D. Dubbers, Phys. Rev. D 38, 2722 (1988).

[19] M. J. Savage, B. W. Filippone, and L. W. Mitchell, Phys. Rev. D 37, 1134 (1988).

[20] M. Bini, T. Fazzini, A. Giannatiempo, G. Poggi, P. Sona, N. Taccetti, K. Elsener, P. G. Hansen, and E. Uggerhøj, Phys. Lett. B 221, 99 (1989). 
[21] T. Asanuma, M. Minowa, T. Tsukamoto, S. Orito, and T. Tsunoda, Phys. Lett. B 237, 588 (1990).

[22] F. W. N. de Boer, J. Lehmann, and J. Steyaert, J. Phys. G 16, L1 (1990).

[23] K. H. Hicks and D. E. Alburger, Phys. Lett. B 276, 423 (1992).

[24] T. Tsunoda, S. Nakamura, S. Orito, and M. Minowa, Europhys. Lett. 30, 273 (1995).

[25] F. W. N. de Boer, R. van Dantzig, J. van Klinken, K. Bethge, H. Bokemeyer, A. Buda, K. A. Müller, and K. E. Stiebing, J. Phys. G 23, L85 (1997).

[26] E. Izaguirre, G. Krnjaic, and M. Pospelov, Phys. Lett. B 740, 61 (2015).

[27] M. Pospelov and Y.-D. Tsai, Phys. Lett. B 785, 288 (2018).

[28] J. Kozaczuk, Phys. Rev. D 97, 015014 (2018).

[29] M. Minowa, Y. Inoue, T. Asanuma, and M. Imamura, Phys. Rev. Lett. 71, 4120 (1993).

[30] A. V. Derbin et al., Pisma Zh. Eksp. Teor. Fiz. 65, 576 (1997) [JETP Lett. 65, 605 (1997)].

[31] A. V. Derbin et al., Yad. Fiz. 65, 1335 (2002) [Phys. At. Nucl. 65, 1302 (2002)].

[32] G. G. Raffelt, Stars as laboratories for fundamental physics (1996).
[33] Y.-S. Liu, D. McKeen, and G. A. Miller, Phys. Rev. Lett. 117, 101801 (2016).

[34] S. Knapen, T. Lin, and K. M. Zurek, Phys. Rev. D 96, 115021 (2017).

[35] M. Hoferichter, P. Klos, J. Menndez, and A. Schwenk, Phys. Rev. Lett. 119, 181803 (2017).

[36] C.-Y. Chen, M. Pospelov, and Y.-M. Zhong, Phys. Rev. D 95, 115005 (2017).

[37] Saint-gobain crystals, https://www.crystals.saint-gobain. com.

[38] S. Agostinelli et al. (GEANT4 Collaboration), Nucl. Instrum. Methods Phys. Res., Sect. A 506, 250 (2003).

[39] M. Agostini, G. Benato, and J. Detwiler, Phys. Rev. D 96, 053001 (2017).

[40] J. C. Loach, J. Cooley, G. A. Cox, Z. Li, K. D. Nguyen, and A. W. P. Poon, Nucl. Instrum. Methods Phys. Res., Sect. A 839, 6 (2016).

[41] Y. Y. Nefedov and P. L. Usenko, Instrum. Exp. Tech. 59, 115 (2016).

[42] P. Yang, C. D. Harmon, F. P. Doty, and J. A. Ohlhausen, IEEE Trans. Nucl. Sci. 61, 1024 (2014).

[43] T. Akesson et al. (LDMX Collaboration), arXiv:1808.05219. 